\title{
Stokes-layer formation under absence of moving parts-A novel oscillatory plasma actuator design for turbulent drag reduction
}

Cite as: Phys. Fluids 31, 051701 (2019); https://doi.org/10.1063/1.5094388

Submitted: 01 March 2019 . Accepted: 25 April 2019. Published Online: 13 May 2019

Marc T. Hehner, Davide Gatti (D), and Jochen Kriegseis

\section{COLLECTIONS}

This paper was selected as Featured

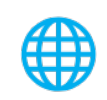

\section{ARTICLES YOU MAY BE INTERESTED IN}

Hypersonic aerodynamic heating over a flared cone with wavy wall

Physics of Fluids 31, 051702 (2019); https://doi.org/10.1063/1.5098543

Geometric optimization of riblet-textured surfaces for drag reduction in laminar boundary layer flows

Physics of Fluids 31, 053601 (2019); https://doi.org/10.1063/1.5090881

Fast flow field prediction over airfoils using deep learning approach

Physics of Fluids 31, 057103 (2019); https://doi.org/10.1063/1.5094943

\section{Qeilioht Highlights of the best new research in the physical sciences}




\title{
Stokes-layer formation under absence of moving parts-A novel oscillatory plasma actuator design for turbulent drag reduction
}

\author{
Cite as: Phys. Fluids 31, 051701 (2019); doi: 10.1063/1.5094388 \\ Submitted: 1 March 2019 - Accepted: 25 April 2019 • \\ Published Online: 13 May 2019
}

Marc T. Hehner, Davide Gatti, (D) and Jochen Kriegseis ${ }^{a)}$

AFFILIATIONS

Karlsruhe Institute of Technology (KIT), Institute of Fluid Mechanics (ISTM), 76131 Karlsruhe, Germany

a)Electronic mail: kriegseis@kit.edu

\begin{abstract}
A novel plasma actuator concept is proposed to mimic the effect of spanwise wall oscillations without mechanically moving parts, where four groups of electrodes and three independently operated high-voltage power supplies maintain a pulsatile dielectric barrier discharge (DBD) array. Time-resolved planar velocity fields are obtained with high-speed particle image velocimetry (PIV) in proximity of the discharge zones for quiescent ambient conditions. Resulting flow topologies and wall-normal velocity profiles indicate the Stokes-layer-like flow formation, which is elevated above the wall due to the no-slip condition. The underlying body forces are derived from the PIV data to provide further insight into cause-effect relations between pulsatile discharge and oscillatory flow. The momentum transfer domain is found to be only interrupted with the width of the exposed electrode, which is an important step toward homogeneous virtual wall oscillations. A comparison with earlier studies by Gatti et al. ["Experimental assessment of spanwise-oscillating dielectric electroactive surfaces for turbulent drag reduction in an air channel flow," Exp. Fluids 56, 110 (2015)] leads to the hypothesis that DBD-based turbulent drag reduction might be a competing alternative to conventional active and passive shear-layer formation strategies, where the adjustability of both oscillation frequency and velocity amplitude might cover a wide range of Reynolds numbers.
\end{abstract}

Published under license by AIP Publishing. https://doi.org/10.1063/1.5094388

The increase of friction drag above the laminar value, one of the fundamental manifestations of turbulent flows, prompts us to study techniques aimed at skin-friction drag reduction (DR) in the turbulent regime. ${ }^{1}$ Of the many ways to achieve this goal-see, e.g., the comprehensive review by Corke and Thomas $^{2}$ - active predetermined techniques ${ }^{3}$ require an action that is not based on the actual flow state and can therefore yield net power savings at the cost of moderate complexity and an energy input.

Streamwise traveling waves (StTW) of spanwise wall velocity ${ }^{4}$ are a successful predetermined near-wall turbulence manipulation, ${ }^{5}$ which achieve sizeable theoretical net-power saving. Their drag-reduction capabilities have been confirmed both numerically ${ }^{4,6,7}$ and experimentally ${ }^{8,9}$ in pipe, channel, and boundary layer flows. Spanwise wall oscillations ${ }^{10}$ by periodic inplane motions of a solid boundary are the simplest StTW. As indicated in Fig. 1, such motions lead to a spanwise shear layer $^{11}$ - the so-called Stokes layer-that favorably interacts with near-wall turbulence up to the buffer layer ${ }^{12}$ and yields drag reduction. ${ }^{13}$

To date, in most experimental implementations, the wall is physically displaced, where the operating range of such approaches is largely limited by mechanical inertia ${ }^{8,15}$ and resonant effects. $9,16,17$ As a notable exception, Ghebali et al. ${ }^{18}$ used a particular undulated surface to passively reproduce the shear profile introduced by StTW for a distinct flow speed.

Plasma actuators (PAs) are active flow-control (AFC) devices that produce a discharge-based body force in the vicinity of the wall, ${ }^{19-21}$ which demonstrated competing AFC properties as compared to conventional actuators. ${ }^{21,22}$ Particularly, the absence of moving parts and the fast response time qualify PAs promising to mimic periodic wall motions by means of oscillatory discharges, as was first demonstrated by Wilkinson. ${ }^{23}$ The variety of different AFC scenarios led to a number of different multielectrode PA arrays, ${ }^{19,2}$ two of which serve as basis for the present novel PA concept for virtual wall oscillations: 


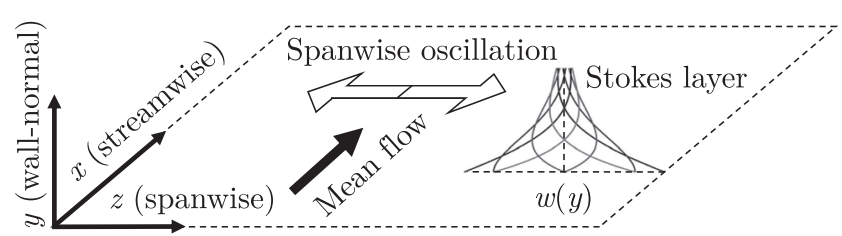

FIG. 1. Sketch of mean flow with wall oscillation: coordinate system, nomenclature, mean flow direction, and resulting stokes layer.

In continuation of earlier reports, ${ }^{23}$ Jukes et al. ${ }^{25}$ mimicked wall oscillations with an array of three-electrode PAs. As indicated in Fig. 2(a), the encapsulated electrode was grounded, whereas the two groups of exposed electrodes were alternately supplied with AC high voltage (HV). The major drawback of this otherwise promising strategy is the large gap between the momentum-transfer domains, which yet remains mandatory for such configurations to prevent from parasitic discharges or coronas between consecutive electrode groups. ${ }^{26}$ Jukes et al., ${ }^{25}$ therefore, suggested to reduce the gap between actuators in the array in order to decrease the spanwise wavelength of the forcing. Such spanwise inhomogeneities have recently been shown to significantly drop the desired effect of drag reduction due to adverse wall normal motions inside the turbulent boundary layer. $^{2}$

Debien et ll $^{28}$ introduced a gapless array of (only) unidirectionally operated actuators [see Fig. 2(b)]. Due to the altered wiring of grounded and HV electrode, the concept minimizes the gap between consecutive discharge zones and to the width of the exposed electrodes at the price of a constant preset momentum-transfer direction.

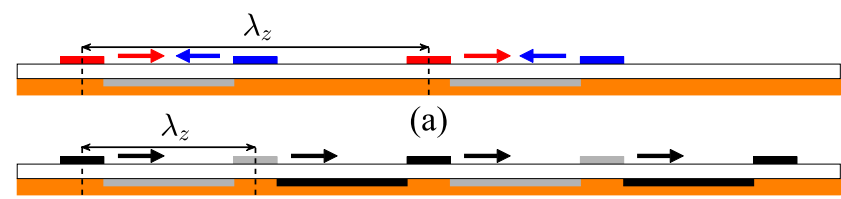

(b)

$\lambda_{z}$

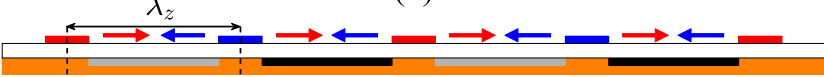

(c)

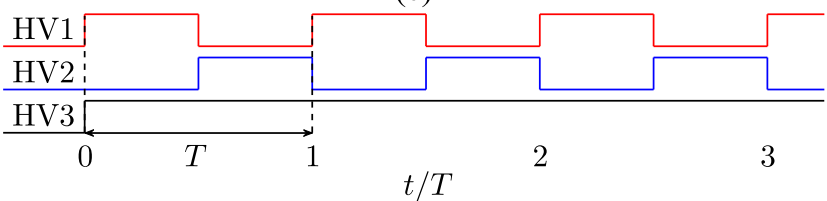

(d)

FIG. 2. Sketch of geometry and electric connection for various PA configurations (not to scale). Color-coded electrodes receive (black solid lines) steady HV operation (HV3) and receive (blue and red solid lines) pulsed HV operation (HV1, HV2, and phase $\Delta \varphi=\pi$ ), gray solid lines are grounded electrodes, white open squares and orange solid lines indicate dielectric material and electrode encapsulation, respectively, and spanwise wavelength $\lambda_{z}$ is added where applicable. (a) Oscillating operation with gap, adapted from Jukes et al., ${ }^{25}$ (b) steady gapless operation, adapted from Debien et al., ${ }^{28}$ (c) novel gapless oscillatory PA concept, and (d) timing diagram for the respective HV transformers in [(a)-(c)]; normalized cycle period $t^{*}=t / T$ is added for clarity.
The novel PA concept centers around the idea to simultaneously take advantages of both above-mentioned approaches, as indicated in Fig. 2(c). Particularly, two groups of exposed electrodes are alternately supplied with HV (HV1: red solid lines and HV2: blue solid lines). Furthermore, the former gap is bridged with a third group of HV electrodes, which is continuously operated (HV3: black solid lines). A fourth group of electrodes remains grounded throughout the PA operation. The hypothesis to significantly reduce the inhomogeneity with the proposed concept is tested on the grounds of time-resolved planar velocity fields in quiescent ambient conditions, where both flow topology and wall-normal velocity profiles of the PA operation are studied in the present letter. Additional remarks comment on the candidating operating range of the present concept and the corresponding achievable amount of drag reduction (DR).

Conductive paint containing micron-sized silver particles was used to spray paint five $1 \mathrm{~mm}$ exposed and four $3 \mathrm{~mm}$ lower electrodes of $10 \mu \mathrm{m}$ thickness on either side of a $500 \mu \mathrm{m}$ thick polyethylene terephthalate (PET) foil; see Yadala et al. ${ }^{29}$ for further details on the computer-controlled fabrication process. A length of $10 \mathrm{~cm}$ was chosen for all electrodes to ensure a quasi-twodimensional flow development-at least in the center region of the PA array. To avoid parasitic discharges below the dielectric, the lower electrodes were further encapsulated with Kapton tape. A GBS ${ }^{30}$ Minipuls 2 continuously supplies the encapsulated HV3 electrode. Two GBS ${ }^{30}$ Minipuls 1 are chosen for the upper electrodes, which operate $\Delta \varphi=\pi$ out of phase with $50 \%$ duty cycle; see Fig. 2(d) for the timing of the operated electrode groups. A duty-cycle frequency of $1 / T=50 \mathrm{~Hz}$ was chosen similar to earlier efforts on wall-oscillation AFC by Gatti et al. ${ }^{17}$ to ensure the comparability of the investigations. All three HV transformers operate at $f_{\mathrm{ac}}=16 \mathrm{kHz}$ and $V_{\mathrm{pp}}=4 \mathrm{kV}$ to maintain the oscillating dielectric barrier discharge (DBD) above all four encapsulated electrodes.

High-speed particle image velocimetry (PIV) experiments are conducted in the immediate vicinity above of the PA array to measure time-resolved plasma-induced velocity fields (see Fig. 3). Note that a coordinate system is added to Fig. 3, which defines $x$ and the main flow $u$ as parallel to the electrodes. Accordingly, $y, v$ and $z, w$ refer to the wall-normal and spanwise components of coordinates and velocities, respectively (cp. also Fig. 1). The flow is seeded with $1 \mu \mathrm{m}$ DEHS particles $\left(\right.$ Stk $\left.=2.3 \times 10^{-3}\right)$, which are illuminated in a wall-normal light sheet $(y, z$-plane) at the PA array center $(x=5 \mathrm{~cm})$ by a Quantronix Darwin-Duo Nd:YLF laser. Two Photron

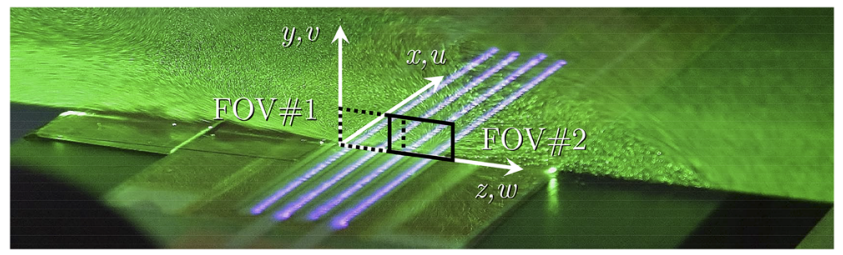

FIG. 3. Operative PA array during PIV experiment. Coordinate system $x, y, z$ and corresponding velocity components $u, v, w$ are indicated; The two FOVs are sketched as solid and dashed rectangles in the light sheet to indicate the recorded flow domain. 
FASTCAM SA 4 cameras (active sensor size $1024 \times 512 \mathrm{px}^{2}, 8$ bits) equipped with Nikon Nikkor $105 \mathrm{~mm}$ microlenses $\left(f_{\#}=8\right)$ were located on either side of the light sheet to capture two fields of view (FOVs) with a $3 \mathrm{~mm}$ overlap and a spatial resolution of $77 \mathrm{px} / \mathrm{mm}$. The merged images cover five exposed electrodes and span four spanwise wavelengths $\left(\lambda_{z}=4 \mathrm{~mm}\right)$. An ILA_5150 synchronizer control unit was used to coordinate double-cavity laser pulses, camera exposures, and HV transformers.

Images were acquired in double-frame mode at a sample rate of $f_{s}=2.4 \mathrm{kHz}$ with a pulse delay of $100 \mu \mathrm{s}$. To ensure quasisteady conditions, the PA array was activated $10 \mathrm{~s}$ before the PIV system obtained data for $4.5 \mathrm{~s}$, hence capturing 225 duty cycles with a phase resolution of 24 bins. PIVview software was used to process the data in a multigrid/multipass approach, where the raw images were cross-correlated on a final interrogation window size of $16 \times 8 \mathrm{px}^{2}$ with an overlap factor of $50 \%$. As such, the resulting velocity information was derived with a spatial resolution of 10 and 20 vectors $/ \mathrm{mm}$ in the $z$ and $y$ direction, respectively. A normalized median test ${ }^{32}$ (threshold 3) was used to replace $2.3 \%$ outliers with the second highest correlation peak. Furthermore, postprocessing of the velocity information and combination of the two FOVs was done with Matlab.

The resulting phase-averaged velocity vector fields are shown in Fig. 4 for the two phases of strongest flow development, i.e., $\varphi=5 \pi / 6$ and $\varphi=11 \pi / 6$. The underlying body force distributions were determined from the PIV data, ${ }^{31,33,34}$ which are shown as contours in Fig. 4. Furthermore, black $10 \%$ isolines ${ }^{31}$ of the force are added to the diagrams to provide immediate insight into the cause-effect relation of the DBD-based virtual wall oscillation. Note that the spanwise coordinate is normalized with the wavelength $\lambda_{z}=4 \mathrm{~mm}$ of the actuator array but additionally appears in physical dimensions to ensure best possible comparability with other DBD PA characterization studies.

At first glance, it is obvious that the momentum transfer domain is only interrupted by the width of the exposed electrode, which is a significant improvement toward the desired homogeneous characteristics of the virtual wall oscillation. ${ }^{25,27}$ The remaining inhomogeneities lead to the formation of vortices in proximity of the $\mathrm{DBD}$, which is expected for unsteady PA operation in quiescent air investigations. ${ }^{35,36}$ The influence of edge-effects due to the limited number of electrodes turned out to be negligible, since the force distributions and developed flow patterns are similar for all four wavelengths $\lambda_{z}$.

To study the effect of the DBD-based virtual wall oscillations more rigorously, the recorded velocity information is spatially averaged onto a single average wavelength $\bar{\lambda}_{z}$ and wall normal profiles of spanwise velocity $w(y)$ are extracted for the considered phase angles $\varphi$ (see Fig. 5). To further study the spatial development of the walljet development, four spanwise locations $z / \bar{\lambda}_{z}$ are distinguished, all of which are indicated in the top left sketch of Fig. 5 to guide the reader.

A direct comparison of Fig. 5(c) and the sketched profiles in Fig. 1 reveals that the velocity profile $w(y)$ above the discharge center at $z / \bar{\lambda}_{z}=0.5$ closely resembles an oscillating Stokes layer. ${ }^{37}$ The main difference occurs due to the no-slip condition at the stationary wall and the resulting wall-jet formation such that the maximum spanwise velocity $w_{\max }$ is slightly elevated above the wall. As expected, the flow above the exposed electrode $\left[z / \bar{\lambda}_{z}=0=1\right.$, Fig. 5(a)] is significantly weaker due to the momentum-transfer inhomogeneity. Furthermore, the footprint of wall-jet formation can be identified from the asymmetric velocity profiles at $z / \bar{\lambda}_{z}=0.25$ and $z / \bar{\lambda}_{z}=0.75$; see Figs. 5(b) and 5(d), respectively. The comparison of the diagrams reveals a range of $0.25 \mathrm{~mm}<y<0.5 \mathrm{~mm}$ for the spanwise peak velocity $\max (w)=W$ of the virtual wall oscillation.

In order to estimate the range of promising flow scenarios and the corresponding flow control success for the novel oscillatory PA concept, the velocity profiles at hand have to be converted to viscous units. ${ }^{2,37}$ However, the wall-normal coordinate $y^{+}=y u_{\tau} / v$ requires knowledge of the friction velocity $u_{\tau}=\sqrt{\tau_{w} / \rho}$ (thus wall shear stress $\left.\tau_{w}\right)$ and the friction Reynolds number $R e_{\tau}=u_{\tau} L / v$ furthermore

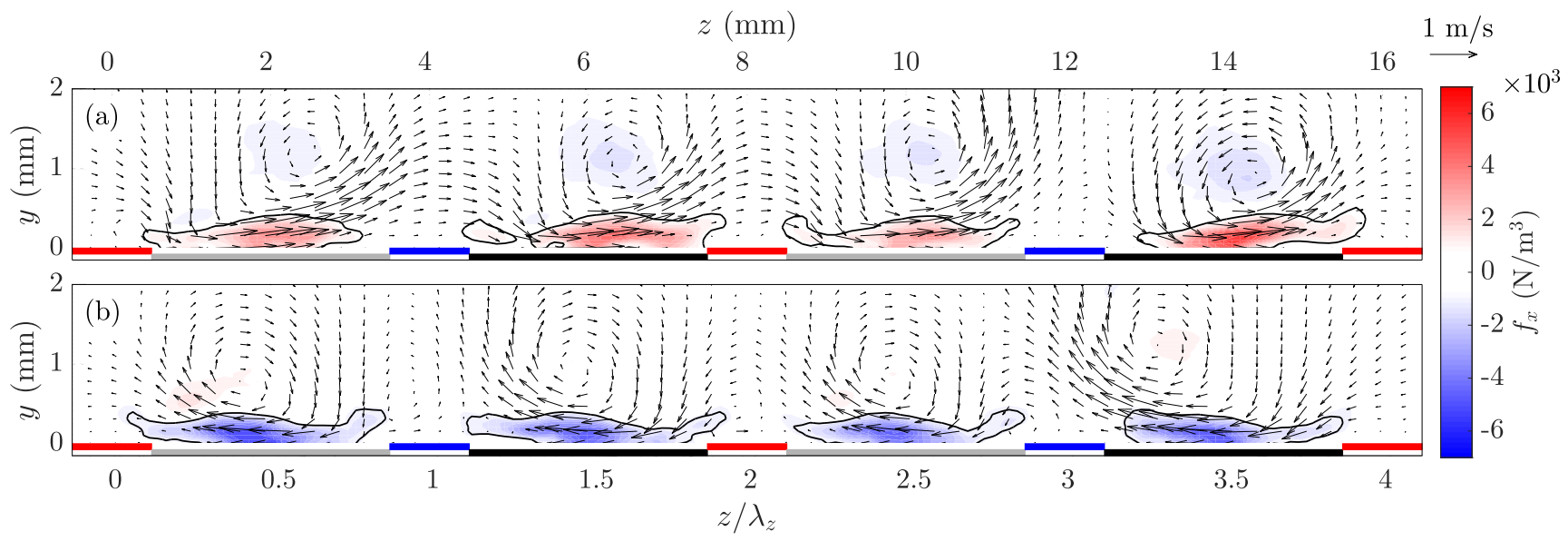

FIG. 4. Phase-resolved velocity fields $\vec{u}(y, z, \varphi)$ (vectors) and underlying body-force densities $f_{z}(y, z, \varphi)$ (contours) for the DBD PA array of wavelengths $\lambda_{z}=4$ mm; upper and lower electrodes are added to indicate discharge locations; note that only every third velocity vector is shown and a black $10 \%$ isoline ${ }^{31}$ of the force is added for clarity. (a) First half cycle $\varphi=5 \pi / 6$, active electrodes: red and black solid lines, (b) second half cycle $\varphi=11 \pi / 6$, active electrodes: blue and black solid lines. 


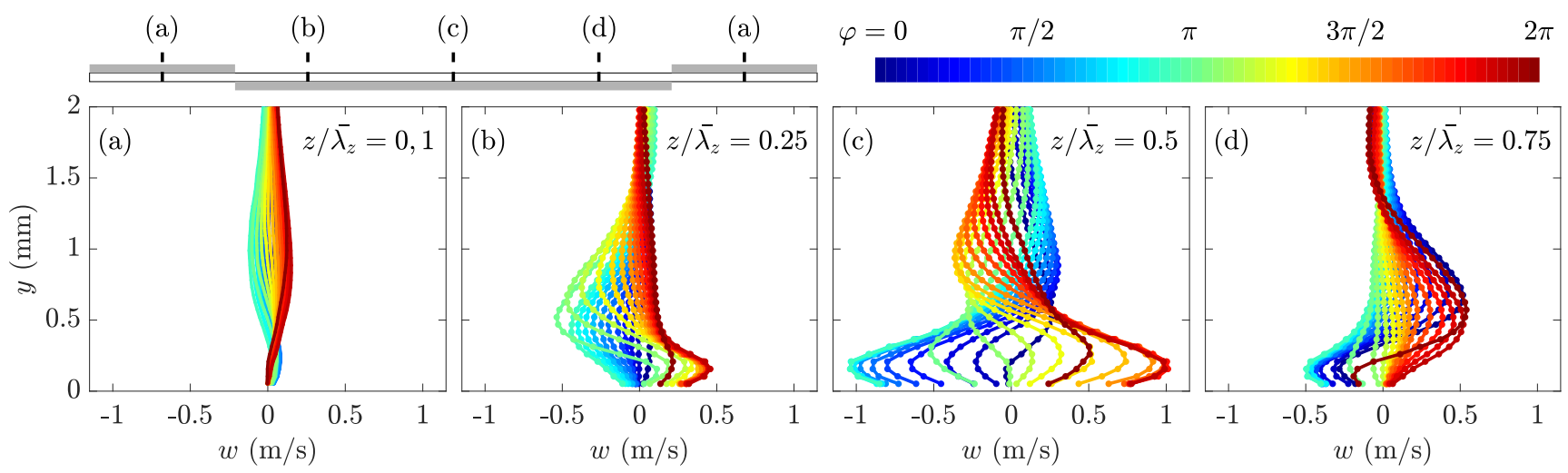

FIG. 5. Wall-normal profiles of spanwise velocity $w(y)$ for various phase angles $\varphi$ and spanwise locations $z / \bar{\lambda}_{z}$; the locations of the phase-averaged velocity profiles of $[(a)-(d)]$ are indicated in the top left sketch of the PA.

contains a characteristic length scale $L$. Therefore, such estimation immediately relies on the considered flow scenario. Since further experiments are scheduled in the same duct flow facility, the flow characteristics obtained in earlier AFC investigations with oscillating elastomer actuators by Gatti et al. ${ }^{17}$ are adopted to illustrate this approach.

Accordingly, a friction velocity $u_{\tau}=0.31 \mathrm{~m} / \mathrm{s}$, duct height $2 H=25.2 \mathrm{~mm}$, and kinematic viscosity $v=1.55 \times 10^{5} \mathrm{~m}^{2} / \mathrm{s}$ lead to a friction Reynolds number of $R e_{\tau}=u_{\tau} H / v=250$ and the above-mentioned range of peak velocities of up to $w^{+}=w / u_{\tau}$ $=3$ fall into the range of $4<y^{+}=y u_{\tau} / v<9$. Based on the correlation of Yakeno et al. ${ }^{13}$ to estimate the drag reduction effect from the rms-profiles of the induced spanwise shear $\partial w^{+} / \partial y^{+}$at $y^{+}=10$ and 15 , the profiles of Fig. 5 are capable of reducing the wall-shear stress by up to $15 \%$. Despite the fact that this number is an upper bound of realistic AFC expectations for the given control concept, this number yet provides evidence that DBD-based turbulent drag reduction might be a competing alternative to conventional active and passive shear-layer formation strategies. Furthermore, it seems promising to also consider different flow scenarios than the above-mentioned ${ }^{17}$ large-aspect-ratio narrow duct flow, as it might lead to higher Re numbers with the same physical actuator parameters; see, e.g., the boundary-layer experiments by Bird et al.

As a final remark, the present work saliently demonstrates the benefits of the additional electrode and the third HV device in order to mimic a Stokes layer without moving parts. It can therefore be concluded that the novel PA concept of virtual wall oscillations is a valuable step toward the replacement of the mechanically moving part for such AFC applications. The major advantage of the DBD-based oscillation is particularly the adjustability of the oscillation frequency and velocity amplitude (via operating voltage) such that optimal AFC can be maintained across a range of Reynolds numbers for both external and internal AFC scenarios.

The authors acknowledge technical support with the Minipuls devices and the actuator fabrication from the PA research groups in Darmstadt and Delft, respectively.

\section{REFERENCES}

${ }^{1}$ J. Kim, "Control of turbulent boundary layers," Phys. Fluids 15, 1093 (2003).

${ }^{2}$ T. C. Corke and F. O. Thomas, "Active and passive turbulent boundary-layer drag reduction," AIAA J. 56, 3835 (2018).

${ }^{3}$ M. Gad-el Hak, Flow Control-Passive, Active and Reactive Flow Management (Cambridge University Press, Cambridge, 2000).

${ }^{4}$ M. Quadrio, P. Ricco, and C. Viotti, "Streamwise-traveling waves of spanwise wall velocity for turbulent drag reduction," J. Fluid Mech. 627, 161 (2009).

${ }^{5} \mathrm{M}$. Quadrio, "Drag reduction in turbulent boundary layers by in-plane wall motion," Philos. Trans. R. Soc., A 369, 1428 (2011).

${ }^{6}$ E. Hurst, Q. Yang, and Y. Chung, "The effect of Reynolds number on turbulent drag reduction by streamwise travelling waves," J. Fluid Mech. 759, 28 (2014).

${ }^{7}$ D. Gatti and M. Quadrio, "Reynolds-number dependence of turbulent skinfriction drag reduction induced by spanwise forcing," J. Fluid Mech. 802, 553 (2016).

${ }^{8}$ F. Auteri, A. Baron, M. Belan, G. Campanardi, and M. Quadrio, "Experimental assessment of drag reduction by traveling waves in a turbulent pipe flow," Phys. Fluids 22, 115103-115114 (2010).

${ }^{9} \mathrm{~J}$. Bird, M. Santer, and J. F. Morrison, "Experimental control of turbulent boundary layers with in-plane travelling waves," Flow, Turbul. Combust. 100, 1015 (2018).

${ }^{10} \mathrm{M}$. Quadrio and P. Ricco, "Critical assessment of turbulent drag reduction through spanwise wall oscillations," J. Fluid Mech. 521, 251 (2004).

${ }^{11}$ M. Quadrio and P. Ricco, "The laminar generalized Stokes layer and turbulent drag reduction," J. Fluid Mech. 667, 135 (2011).

${ }^{12}$ S. Pope, Turbulent Flows (Cambridge University Press, Cambridge, 2000).

${ }^{13}$ A. Yakeno, Y. Hasegawa, and N. Kasagi, "Modification of quasi-streamwise vortical structure in a drag-reduced turbulent channel flow with spanwise wall oscillation," Phys. Fluids 26, 085109 (2014).

${ }^{14} \mathrm{~L}$. Agostini, E. Touber, and M. A. Leschziner, "The turbulence vorticity as a window to the physics of friction-drag reduction by oscillatory wall motion," Int. J. Heat Fluid Flow 51, 3 (2015).

${ }^{15}$ K.-S. Choi and B. R. Clayton, "The mechanism of turbulent drag reduction with wall oscillation," Int. J. Heat Fluid Flow 22, 1 (2001).

${ }^{16}$ K. Gouder, M. Potter, and J. Morrison, "Turbulent friction drag reduction using electroactive polymer and electromagnetically driven surfaces," Exp. Fluids 54, 1441 (2013).

${ }^{17}$ D. Gatti, A. Güttler, B. Frohnapfel, and C. Tropea, "Experimental assessment of spanwise-oscillating dielectric electroactive surfaces for turbulent drag reduction in an air channel flow," Exp. Fluids 56, 110 (2015). 
${ }^{18}$ S. Ghebali, S. I. Chernyshenko, and M. A. Leschziner, "Can large-scale oblique undulations on a solid wall reduce the turbulent drag?," Phys. Fluids 29, 105102 (2017).

${ }^{19} \mathrm{~N}$. Benard and E. Moreau, "Electrical and mechanical characteristics of surface AC dielectric barrier discharge plasma actuators applied to airflow control," Exp. Fluids 55, 1846 (2014).

${ }^{20} \mathrm{M}$. Kotsonis, "Diagnostics for characterisation of plasma actuators," Meas. Sci. Technol. 26, 092001 (2015).

${ }^{21}$ J. Kriegseis, B. Simon, and S. Grundmann, "Towards in-flight applications? A review on dielectric barrier discharge-based boundary-layer control," Appl, Mech. Rev. 68, 020802 (2016).

${ }^{22}$ L. N. Cattafesta and M. Sheplak, "Actuators for active flow control," Annu. Rev. Fluid. Mech. 43, 247 (2011).

${ }^{23}$ S. P. Wilkinson, "Investigation of an oscillating surface plasma for turbulent drag reduction," AIAA Paper 2003-1023, 2003.

${ }^{24}$ K.-S. Choi, T. Jukes, and R. Whalley, "Turbulent boundary-layer control with plasma actuators," Philos. Trans. R. Soc., A 369, 1443 (2011).

${ }^{25}$ T. Jukes, K.-S. Choi, G. Johnson, and S. Scott, "Turbulent drag reduction by surface plasma through spanwise flow oscillation," AIAA Paper 2006-3693, 2006.

${ }^{26}$ M. Forte, J. Jolibois, J. Pons, E. Moreau, G. Touchard, and M. Cazalens, "Optimization of a dielectric barrier discharge actuator by stationary and non-stationary measurements of the induced flow velocity: Application to airflow control," Exp. Fluids 43, 917 (2007).

${ }^{27}$ S. Straub, R. Vinuesa, P. Schlatter, B. Frohnapfel, and D. Gatti, "Turbulent duct flow controlled with spanwise wall Oscillations," Flow, Turbul. Combust. 99, 787 (2017).
${ }^{28}$ A. Debien, N. Benard, and E. Moreau, "Streamer inhibition for improving force and electric wind produced by DBD actuators," J. Phys. D: Appl. Phys. 45, 215201 (2012).

${ }^{29}$ S. Yadala, M. T. Hehner, J. Serpieri, N. Benard, P. C. Dörr, M. J. Kloker, and M. Kotsonis, "Experimental control of swept-wing transition through base-flow modification by plasma actuators," J. Fluid Mech. 844, R2 (2018).

${ }^{30}$ See https://www.gbs-elektronik.de/en/ for information about the Minipuls HV transformers.

${ }^{31}$ J. Kriegseis, C. Schwarz, C. Tropea, and S. Grundmann, "Velocity-informationbased force-term estimation of dielectric-barrier discharge plasma actuators," J. Phys. D: Appl. Phys. 46, 055202 (2013).

${ }^{32} \mathrm{~J}$. Westerweel and F. Scarano, "Universal outlier detection for PIV data," Exp. Fluids 39, 1096 (2005).

${ }^{33}$ M. Kuhnhenn, B. Simon, I. Maden, and J. Kriegseis, "Interrelation of phaseaveraged volume force and capacitance of dielectric barrier discharge plasma actuators," J. Fluid Mech. 809, R1 (2016).

${ }^{34} \mathrm{~N}$. Benard, A. Debien, and E. Moreau, "Time-dependent volume force produced by a non-thermal plasma actuator from experimental velocity field," J. Phys. D: Appl. Phys. 46, 245201 (2013).

${ }^{35}$ P. Sattari, D. E. Rival, R. J. Martinuzzi, and C. Tropea, "Growth and separation of a start-up vortex from a two-dimensional shear layer," Phys. Fluids 24, 107102 (2012).

${ }^{36} \mathrm{R}$. Whalley and K.-S. Choi, "Starting, traveling, and colliding vortices: Dielectric-barrier-discharge plasma in quiescent air," Phys. Fluids 22, 091105 (2010).

${ }^{37}$ H. Schlichting and K. Gersten, Boundary-Layer Theory, 9 th ed. (Springer Berlin Heidelberg, 2017). 